\title{
Neuralgia essencial do nervo trigêmeo. Análise de 105 casos
}

\author{
Clement Hamani ${ }^{*}$ Pedro Paulo Mariani Lima Santos*, José Cláudio Marinho da \\ Nóbrega*, Pedro Padilha de Menezes*, Valter Ângelo Sperling Cescato*, Manoel \\ Jacobsen Teixeira** \\ Centro Interdisciplinar de Dor do Hospital das Clínicas da Faculdade de Medicina da Universidade de São Paulo
}

\section{RESUMO}

Os autores analisam seqüencialmente os aspectos clínicos e terapêuticos de 105 doentes com neuralgia do trigêmeo. A média de idades foi de 61,6 anos; $64,8 \%$ dos doentes eram do sexo feminino. A dor foi descrita, mais freqüentemente, como choque de curta duração, esteve principalmente localizada no território da segunda divisão do nervo trigêmeo e o mecanismogatilho mais comum foi a mastigação. O tratamento medicamentoso em todos os casos constituiu no uso da carbamazepina em doses crescentes. Quando ocorreram efeitos indesejáveis, a fenitoína foi a droga substituta em $7,1 \%$ dos casos. Apesar de ter ocorrido melhora inicial na maioria dos casos, 40 (47\%) doentes necessitaram de tratamento cirúrgico: a rizotomia percutânea mecânica com balão do nervo trigêmeo foi o único procedimento em 95,9\% desses casos; a rizotomia percutânea por radiofreqüência foi necessária em 4,1\% dos casos, todos refratários à compressão. Todos os doentes apresentaram melhora significativa da sintomatologia após a execução dos procedimentos operatórios.

\section{PALAVRAS-CHAVE}

Neuralgia do trigêmeo. Dor facial. Rizotomia do trigêmeo.

\section{ABSTRACT}

Essential trigeminal neuralgia. Analysis of 105 cases

The authors present their 105 consecutive cases of trigeminal neuralgia. The mean age of the patients was 61.6 years and $64.5 \%$ of the patients were female. Short duration shock-like episodes were the most common sensory presentation of the pain; the territory of the second division of trigeminal nerve was the region most frequently affected; mastication was the most common trigger mechanism.

Increasing doses of carbamazepine were prescribed to all patients; phenytoin was prescribed to $7.1 \%$ of the cases due to side effects with carbamazepine. Despite of adequate initial response to these drugs in the majority of the cases, 40 (47\%) patients needed to be submitted to neurosurgical treatment: Gasseriam ganglion microcompression by baloon was the procedure of choice in all of the patients of this series. Microcompression was not effective in $4.1 \%$ who underwent trigeminal radiofrequency rhizotomy afterwards. All patients surgically treated presented important relief of pain.

\section{KEYWORDS}

Trigeminal neuralgia. Facial pain. Trigeminal rhizotomy.

\section{INTRODUÇÃO}

É provável que a neuralgia do trigêmeo tenha afetado a humanidade muito tempo antes de ser reconhecida como entidade clínica definida. Segundo
Meyrowsky e Pipito $^{63}$ e Stookey e Ransohoff ${ }^{96}$, foi mencionada pela primeira vez por volta do ano 400 a.C., mas foram Arataeus e Galeno que, ulteriormente, fizeram as primeiras referências mais completas dessa condição, seguidos, segundo Ameli ${ }^{3}$, durante a Idade

\footnotetext{
* Médicos do Centro Interdisciplinar de Dor do HCFMUSP.

** Chefe do Centro Interdisciplinar de Dor e Diretor da Divisão de Neurocirurgia Funcional do HCFMUSP.
} 
Média, por Avicenna (980-1030) e Jurjani (1066-1136). A primeira descrição clara da doença foi elaborada por Massa, em 1550, durante o Renascimento ${ }^{76}$, por John Locke durante o Iluminismo, em $1677^{96}$, e Johannes Lauretntius Baush em $1688^{63}$. Foi reconhecida como entidade definida em 1756 por André, e Sir John Fothergill, em 1773, fez a primeira descrição clássica da neuralgia do trigêmeo ${ }^{76,96}$. A identificação anatômica do nervo trigêmeo, por Bell, em 1821 e 1829, permitiu o desenvolvimento da terapêutica cirúrgica das neuralgias da face, que ocorreu a partir da segunda metade do século XIX ${ }^{96}$.

Poucos relatos epidemiológicos foram publicados sobre a neuralgia do trigêmeo. Manifesta-se em 155 de cada 1 milhão de habitantes, pelo menos, no hemisfério norte ${ }^{7,13}$, onde apresenta incidência anual de 4,3 casos novos por 100 mil habitantes ${ }^{121}$, ou seja, cinco casos novos ao ano por 100 mil mulheres ou 2,7 casos novos ao ano por 100 mil homens.

Os sintomas da neuralgia essencial do trigêmeo são padronizados e inconfundíveis. As descrições clínicas originais, realizadas por Locke em 1677, André em 1756, Fothergill em 1773 e Pujol em 1787, coincidem com as dos autores modernos ${ }^{96}$. Apesar de as apresentações serem padronizadas, as considerações sobre a apresentação clínica da neuralgia do trigêmeo têm sido objeto de poucas publicações nos anos recentes, o que motivou a realização deste estudo, em que foram considerados alguns aspectos clínicos e terapêuticos referentes a doentes com essa afecção, tratados seqüencialmente no Ambulatório do Centro Interdisciplinar de Dor do Hospital das Clínicas da Faculdade de Medicina da Universidade de São Paulo.

\section{Casuística e métodos}

Foram analisados prospectivamente os dados de 105 doentes com queixas clínicas compatíveis com neuralgia do trigêmeo, acompanhados no Centro Interdisciplinar de Dor do Hospital das Clínicas da Faculdade de Medicina da Universidade de São Paulo, durante o período entre 1996 e 2000. Todos foram avaliados em sala privativa do ambulatório por neurocirurgião especializado no tratamento de neuralgias, segundo protocolo padronizado para avaliar algias da face, no qual constam informações sobre idade, sexo, duração das queixas, natureza da dor, território trigeminal acometido, evolução da doença, fenômenos associados, fatores desencadeantes, intensidade da dor, medicamentos analgésicos utilizados, duração e resultado dos tratamentos instituídos, natureza dos procedimentos cirúrgicos necessários e seus resultados.
Tomografia computadorizada do crânio (TC) foi realizada em todos os casos.

\section{Resultados}

As idades variaram de 33 a 84 anos; a média de idades foi de 61,6 anos. Em 56\% dos doentes a idade variou de 60 a 80 anos. Ocorreu predomínio dos doentes do sexo feminino; $68(64,8 \%)$.

A dor apresentava uma ou mais características: foi descrita como sensação de choque de curta duração por $69,5 \%$ dos doentes; pontadas e/ou agulhadas por $40 \%$ dos doentes; queimor por $35,2 \%$; ou latejamento por $20 \%$. Cerca de $40 \%$ dos doentes não foram capazes de precisar o padrão da dor.

A dor era paroxística, sendo de curta duração em $55,3 \%$ dos doentes; de longa duração em $21,9 \%$; constante com episódios de exacerbação em 15,2\%. Em $62 \%$ dos doentes a dor ocorreu do lado direito da face e, nos demais, à esquerda.

$\mathrm{O}$ território mais acometido foi a segunda divisão ou V2 (31,4\%), seguido-se o V3 (26,7\%), V2 e V3 (22,8\%), V1 eV2 (10,5\%), V1,V2 e V3 (4,8\%) e V1 (3,8\%).

Em cerca de $45 \%$ dos doentes, pelo menos em algum período da doença, a dor apresentou intensidade $10-$ segundo a escala analógica numérica de 0 a $10-$, achado que traduz o sofrimento e a incapacidade dela decorrente.

Mecanismos ou zonas de gatilho que acionados deflagravam a dor foram evidenciados em todos os doentes. Os mais comuns foram: mastigação $(61,9 \%)$, fala $(47,6 \%)$, contato físico em algum ponto do tegumento do território acometido $(37,1 \%)$ e/ou frio $(26,7 \%)$. Associados à dor, alguns doentes queixavam-se de sensação de queimor $(20,9 \%)$, dormência $(18,1 \%)$ e/ou de lacrimejamento $(17,1 \%)$.

$\mathrm{O}$ exame neurológico não revelou anormalidades da função trigeminal. Em todos os casos, a tomografia computadorizada não revelou anormalidades relacionadas às vias trigeminais, mas apenas alterações próprias das faixas etárias a que pertenciam os doentes.

O tratamento medicamentoso consistiu do uso de carbamazepina em todos os casos, em dose crescente, até haver controle da dor ou ocorrência de efeitos colaterais. Em 35,7\% dos casos, a carbamazepina foi associada à fenitoína, porque, isoladamente, não havia sido eficaz. Em 7,1\% dos casos, os efeitos indesejáveis decorrentes do uso da carbamazepina justificaram sua substituição por fenitoína. Em 40 (47,0\%) doentes não houve controle da dor com a máxima dosagem tolerada desses medicamentos e, nestes, o tratamento cirúrgico foi realizado. A rizotomia percutânea por compressão 
com balão do gânglio trigeminal foi empregada em 95,9\% dos casos e a rizotomia percutânea por radiofreqüência, em 4,1\%, quando a compressão com balão não foi eficaz. Após as cirurgias, a dor foi eliminada totalmente em $36(91,5 \%)$ casos. Nos 4 (8,5\% dos operados) doentes restantes, houve recorrência da dor e nova operação foi proposta.

\section{Discussão}

Segundo White e Sweet ${ }^{119}$, a neuralgia do trigêmeo caracteriza-se por cinco aspectos básicos: é paroxística, apresenta fatores desencadeantes definidos, limita-se ao território de distribuição do nervo trigêmeo, acomete um lado da face e não se associa a anormalidades ao exame neurológico. Klingher ${ }^{58}$ enfatiza a ausência de causas orgânicas evidentes e a resposta terapêutica favorável à secção dos troncos do nervo ou da raiz trigeminal, e Penman ${ }^{75}$ considerou ser a intensidade da dor aspecto importante para o diagnóstico, mas, em alguns casos, discretas anormalidades sensitivas podem ser evidenciadas no território de distribuição do nervo trigêmeo ${ }^{120}$; quando o exame físico é mais detalhado, anormalidades sensitivas podem ser evidenciadas em até um terço dos casos ${ }^{52,62,75}$.

$\mathrm{Na}$ presente casuística, a dor foi considerada intensa, sendo-lhe atribuída o valor 10 da escala visual analógica por $45 \%$ dos doentes. Foi descrita como choque e paroxística em todos os casos, o que está de acordo com o descrito por outros autores que similarmente a descreveram como tal ou como sensação de sucessivas pontadas, facadas, queimor, relâmpagos ou penetração de calor de forte intensidade na face f $^{29,42,75,119}$ que, ao ceder, não deixa desconforto residual. Na presente casuística, alguns doentes queixavam-se de sensação de queimor $(20,9 \%)$ ou dormência $(18,1 \%)$ associada à dor; admite-se que sensação de queimor de curta duração possa ocorrer na fase pós-crítica ${ }^{42}$, principalmente após crises prolongadas ${ }^{51}$. Raramente o doente apresenta dor menos intensa no intervalo intercrítico ${ }^{52,75,96}$. Instala-se e desaparece subitamente, tem curta duração e reaparece em intervalos variados. Cada surto pode ter a duração de dias, semanas ou meses ${ }^{40,96}$. A freqüência das crises costuma ser fixa ou varia de modo padronizado ${ }^{75}$. Quando as crises são muito freqüentes, o doente pode descrevê-las como contínuas. Os períodos de acalmia podem durar vários dias, semanas, meses ou anos ${ }^{42}$.

$\mathrm{Na}$ presente casuística, manifestou-se nos indivíduos adultos e predominou ( $51,6 \%$ dos casos) na faixa etária de 60 a 90 anos. A neuralgia do trigêmeo raramente ocorre em doentes jovens. A média etária dos doentes, no início da sintomatologia, situa-se entre a sexta e oitava década na maioria das casuísticas ${ }^{2,81,84,121}$. Em dois terços dos doentes de Yoshimasu e cols. ${ }^{121}$, a dor teve início entre 50 e 69 anos de idade. Na maioria das casuísticas, são poucos os doentes com neuralgia do trigêmeo com idade inferior a 30 anos $^{42,96,121}$. Na casuística de Rushton e MacDonald ${ }^{83}$, a idade era inferior a 40 anos em somente $12,3 \%$ dos doentes; na de Henderson $^{42}, 1 \%$ dos doentes relatou que a dor teve início entre 16 a 29 anos de idade.

Na presente casuística, foi mais comum no sexo feminino $(64,8 \%)$, coincidindo com o observado por outros autores, que relataram $51 \%$ a $66 \%$ dos doentes do sexo feminino ${ }^{11,39,47,82,96}$.

$\mathrm{Na}$ presente casuística, a dor predominou à direita $(62 \%)$, o que coincide com o observado em outros trabalhos, em que $50 \%$ a $62,2 \%$ dos doentes apresentaram o lado direito acometido ${ }^{11,39,47,82,96,121}$. Aneuralgia do trigêmeo é bilateral em 0,3\% a 7,07\% dos $\operatorname{casos}^{20,23,28,32,39,47,74,75,82,121}$. Excepcionalmente, a dor inicia-se concomitantemente em ambos os $\operatorname{lados}^{35,83,96,119}$. Na maioria das vezes, a dor contralateral inicia-se alguns anos após o início do quadro $^{42,76}$. O comprometimento de territórios simétricos é freqüente, principalmente quando a divisão mandibular é a afetada ${ }^{42}$. Em $82,6 \%$ a $83 \%$ das vezes, a neuralgia bilateral ocorre nos indivíduos do sexo feminino ${ }^{28,39}$.

A dor limita-se à área inervada por uma ou mais divisões do nervo trigêmeo e não se irradia para os limites periféricos do território comprometido, a não ser quando muito intensa ${ }^{47}$. Na presente casuística, localizou-se predominantemente em V2 e V3, o que também correspondeu com o relato de outros autores $^{39,42,121}$. Ocorre mais freqüentemente na segunda e terceira divisões do nervo e, raramente, na primeira ${ }^{42,119}$. Muitas vezes, mais de uma divisão está envolvida. É muito raro o comprometimento concomitante da primeira e terceira divisões, poupando a segunda. A dor acometeu isoladamente ou associadamente outras divisões em $18,1 \%$ dos doentes da presente casuística. A primeira divisão é sede da dor em $2 \%$ a $7 \%$ dos $\operatorname{casos}^{42,83,96,119}$. A dor da terceira divisão ocorre no lábio inferior e na gengiva e, raramente, na língua ${ }^{96}$, e a da segunda divisão irradia-se para o lábio superior, a asa do nariz, a bochecha e, raramente, para a gengiva e o palato ${ }^{119}$. Somente quando ocorre sobre o globo ocular, na órbita, na região supra-orbitária ou no fronte pode-se afirmar que a divisão oftálmica está envolvida ${ }^{96}$. Em algumas circunstâncias, a dor irradia-se para fora da área de distribuição do nervo trigêmeo. Às vezes, origina-se fora do território trigeminal, o que pode dificultar o diagnóstico ${ }^{27,39,96}$.

A dor costuma ser agravada por estímulos externos, tácteis ou proprioceptivos, aplicados em certas regiões da face ou de outras regiões do corpo ${ }^{96}$. Na presente casuística, a dor era desencadeada por fenômenos 
físicos em todos os doentes, que foram representados principalmente por mastigação $(61,9 \%)$, fala $(47,6 \%)$, contato físico em algum ponto do tegumento do território acometido (37,1\%) e/ou frio (26,7\%). As regiões de excitabilidade aumentada são denominadas zonas de gatilho ou de zonas algiogênicas ${ }^{73}$. São pequenas áreas onde estímulos tácteis de fraca intensidade desencadeiam dor intens $\mathrm{a}^{59}$. Geralmente restringem-se a uma pequena região ou a um ponto localizado nas porções mediais da face ${ }^{59,96}$. Os estímulos tácteis ou proprioceptivos, mas não os dolorosos, térmicos ou pressóricos profundos, desencadeiam a dor. Os receptores de adaptação rápida presentes nas zonas de gatilho são responsáveis pela ocorrência da crise. Ocorrência de zona de gatilho na hemiface oposta é sinal de que dor contralateral deverá surgir no futuro. A zona de gatilho está presente em mais de $50 \%$ dos $\operatorname{casos}^{59,96,121}$. É detectada em $55 \%$ das vezes nas neuralgias da segunda divisão, em $11 \%$ da primeira, em $11 \%$ da terceira e em $22 \%$ quando há combinação de divisões ${ }^{121}$. Localiza-se mais comumente em sulco nasolabial, lábio superior, bochecha, asa do nariz, ângulo da boca e gengiva alveolar ou vestibular ${ }^{59}$.

Fenômenos neurovegetativos representados por lacrimejamento foram relatados por $17,1 \%$ dos doentes na presente casuística. Salivação, lacrimejamento, rinorréia, congestão da mucosa nasal, hiperemia cutânea e edema da face podem acompanhar a manifestação álgica ${ }^{41,75}$. Os fenômenos vasomotores e secretórios foram observados em $30 \%$ dos casos de Forjaz ${ }^{27}$. Raramente, síndrome de Claude Bernard-Horner transitória acompanha a crise ${ }^{1}$. Tais anormalidades podem tornar o diagnóstico diferencial difícil com outras afecções, como cefaléia em salvas, cefaléia do tipo SUNCT, etc. Durante a crise, pode haver contração clônica ou espasmo da hemiface homolatera ${ }^{96}$, o que não ocorreu com os doentes da presente casuística.

Muitas condições álgicas que acometem a face podem assemelhar-se à neuralgia essencial do nervo trigêmeo, incluindo a neuralgia trigeminal atípica, outras neuralgias essenciais da face, como a neuralgia do nervo glossofarínge ${ }^{104}$, a dor facial atípica ${ }^{108}$, a dor facial sintomática decorrente de processos expansivos do gânglio de Gasser ou da raiz do nervo trigêmeo ${ }^{104,108}$, a neuralgia pós-herpética trigeminal ${ }^{96}$, a neuropatia trigeminal essencial ${ }^{38}$, as anormalidades funcionais e estruturais da articulação temporomandibular ${ }^{25}$, síndrome estilóide-estilomastóidea ${ }^{107}$, a distrofia simpaticorreflexa da face ${ }^{104}$, a cefaléia em salvas ${ }^{119}$, as odontalgias $^{108}$, os processos inflamatórios e infecciosos das estruturas profundas da face ${ }^{104,}$, a síndrome paratrigeminal de Raeder ${ }^{116}$ e as afecções do sistema nervoso central (SNC) ${ }^{88}$. Muitos admitem que a neuralgia do trigêmeo seja sempre condição sintomática e decorrente de anormalidades sediadas na "zona de entrada" da raiz trigeminal; Dandy ${ }^{24}$ observou compressão da raiz por veias em $14 \%$ dos seus casos e Gardner e Miklos ${ }^{33}$, alças vasculares anômalas acoladas à raiz trigeminal. Placa de esclerose múltipla na zona de entrada também é causa de neuralgia com características essenciais ${ }^{60}$. Gonzalez-Revilla ${ }^{34}$ revisou 473 casos em que a craniectomia de fossa posterior foi indicada para o tratamento de neuralgia do trigêmeo e observou, em 5,1\% deles, lesões expansivas representadas por neurinoma do nervo acústico, cistos epidermóides ou meningeomas de fossa posterior. Em $1,2 \%$ dos 250 doentes com neuralgia do trigêmeo e com exame neurológico normal, avaliados por Walker ${ }^{117}$, havia processo expansivo no ângulo pontocerebelar. Van Loveren ${ }^{115}$ avaliou mil doentes com dor facial; encontrou lesões expansivas na fossa posterior, representadas por neurinoma do trigêmeo e de acústico, metástase ou meningeoma em 11. Presentemente, a explicação mais razoável para neuralgia do trigêmeo é a que admite existência de etiologia periférica e patogênese central ${ }^{6,54}$. A neuralgia ocorreria em indivíduos com prévia suscetibilidade, quando alguma doença ou irritação periférica do nervo geraria condições para ocorrência de potenciais ectópicos que sensibilizariam os neurônios do núcleo trigeminal ${ }^{30}$. Lesões centrais minúsculas modificariam as condições neurofisiológicas de percepção da dor. Essas alterações facilitariam a deflagração de descargas paroxísticas que, por alguma anormalidade, como a causada pela compressão nervosa por alças vasculares, é capaz de converter potenciais incipientes em potenciais de ação com manifestação clínica e eletrofisiológica ${ }^{56}$. Portanto, mesmo quando não há anormalidades ao exame neurológico em doentes com neuralgia do trigêmeo ${ }^{96}$, exames de imagem devem ser sempre realizados. $\mathrm{Na}$ presente casuística, a TC foi realizada em todos os casos e não revelou anormalidades causais relacionadas às vias trigeminais em nenhum doente. Naidich e cols. ${ }^{69}$ concluíram que a tomografia permite o diagnóstico em $83 \%$ dos casos de neurinoma do acústico, em $90 \%$ de meningeomas da fossa posterior e em $100 \%$ de colesteatomas e aneurismas. A tomografia pode, entretanto, ser normal em doentes com neurinomas do trigêmeo ${ }^{61}$ ou com neurinomas do nervo acústico $^{51}$ de pequenas dimensões. $\mathrm{O}$ estudo angiográfico é indicado para o diagnóstico de má-formações vasculares acometendo as vias trigêminais ${ }^{104}$. Segundo Hutchins e cols. ${ }^{49}$, a ressonância magnética (RM) é o método de imagem de eleição para avaliação da neuralgia do trigêmeo, sendo superior à TC para avaliar a anatomia do tronco encefálico, tais como placas de esclerose múltipla, gliomas do tronco encefálico, acidentes vasculares, vasos anômalos comprimindo a zona 
de entrada da raiz do nervo trigêmeo e tumores cisternais. O exame do líquido cefalorraquidiano é indicado em casos suspeitos de neuropatia trigeminal essencial ou sintomática, quando há suspeita de carcinomatose meníngea ${ }^{104}$. Stöhr e cols.$^{95}$ observaram que, em $41 \%$ dos doentes com neuralgia do trigêmeo, a latência do primeiro componente positivo do potencial somatossensorial é prolongada quando a estimulação é realizada no território da segunda e terceira divisões do nervo. Em casos de neuropatia trigeminal, recomenda-se a pesquisa de reações séricas inflamatórias, os testes para pesquisa de colagenoses, as reações sorológicas para sífilis, o exame do líquido cefalorraquidiano, os estudos eletrofisiológicos e neurorradiológicos ${ }^{87}$. Os exames complementares devem ser repetidos, mesmo quando normais, em casos de neuropatia trigeminal ${ }^{112}$. As alterações podem ocorrer, muitas vezes, muito tempo após o início da doença ${ }^{48,104}$.

Qualquer que seja a etiologia da dor, o processo patológico deve progredir, porque a história natural da neuralgia do trigêmeo demonstra que as crises tendem a ser mais freqüentes e mais prolongadas com o passar do tempo ${ }^{54}$. Vários agentes neurotóxicos foram utilizados para tratar a neuralgia do trigêmeo, incluindo o tricloroetileno ${ }^{83}$ e a estilbamidina ${ }^{96}$. Segundo Braham e Saia $^{10}$, Bergoignan, em 1942, empregou pela primeira vez a difenil-hidantoína no tratamento da neuralgia do trigêmeo. A partir daí, este agente passou a ser amplamente utilizado para tal fim, pois proporciona melhora clínica inicial em $54 \%$ a $70 \%$ dos doentes ${ }^{8,9,10}$. Vertigens são observadas em $61 \%$ dos doentes que utilizam esse fármaco, sendo intoleráveis em 14\%. A carbamazepina foi pela primeira vez utilizada no tratamento da neuralgia do trigêmeo por Blom, em 1962 considerada o medicamento mais eficaz para o tratamento dessa condição ${ }^{12}$. Os resultados iniciais são excelentes em $40 \%$ a $100 \%$ dos doentes $5,9,12,16,55,77,79,81$. A melhora inicial mantém-se em $81 \%$ dos casos durante seis meses e em mais $7 \%$ a $10 \%$ dos casos quando associada à difenil-hidantoína ${ }^{9,66}$. A melhora ocorre nas primeiras 24 horas em $74 \%$ a $85 \%$ dos $\operatorname{casos}^{79,81}$. A dor recorre 24 horas após a suspensão do tratamento ${ }^{81}$. Segundo Motta e cols. ${ }^{66}$, a maioria dos doentes é beneficiada a longo prazo, mas, em $15 \%$ dos casos, há efeitos colaterais que implicam a suspensão do tratamento; na dose de 600 $\mathrm{mg} / \mathrm{dia}, 43 \%$ dos doentes apresentam vertigens e $50 \%$, sonolência e limitação das atividades diárias ${ }^{55}$. A dose necessária para controlar a dor causa vertigem em 10\% dos doentes, sonolência transitória em 7,5\% e exantema em $2,5 \%{ }^{47}$. O clonazepam proporciona alívio em $22 \%$ a $23,3 \%$ dos casos, mesmo quando a dor é resistente à carbamazepina $^{17,93}$, mas à custa de sonolência em $88 \%$ dos doentes, instabilidade da marcha em $80 \%$ e con- fusão mental em $8 \%{ }^{17}$. Outros anticonvulsivantes, como a oxicarbazepina, o valproato de sódio, o ácido valpróico, o divalproato, a gabapentina, o topiramato, a lamotrigina e a vigabatrina são também eficazes. Fromm e cols. ${ }^{31}$ observaram que houve melhora clínica persistente em 10 de 14 doentes com neuralgia do trigêmeo tratados com o baclofeno. Sonolência, sensação de fraqueza, náuseas e vômitos são os principais efeitos colaterais da droga. A associação com a carbamazepina melhora o resultado ${ }^{22}$. A lidocaína, administrada parenteralmente, controla crises de dor trigeminal ${ }^{59}$. Há evidências de que doentes tratados com antidepressivos tricíclicos, neurolépticos (pimozida), capsaicina, propranolol e aplicação tópica de anestésicos locais (EMLA) também podem ser parcialmente beneficiados ${ }^{108}$.

Com o passar do tempo, entretanto, em considerável número de casos, a dor costuma tornar-se rebelde; o aumento de dose pode resultar em intolerância farmacológica. Em $47 \%$ dos casos tratados, observamos, como relatado por outros autores, que os doentes, apesar de terem apresentado melhora inicial, passaram a apresentar dor rebelde, de modo que o tratamento neurocirúrgico foi necessário. A rizotomia percutânea com balão foi a primeira intervenção proposta, pois trata-se de método simples, seguro e eficaz ${ }^{14,67,102}$; a rizotomia percutânea por radiofreqüência foi indicada apenas nos casos em que a rizotomia com balão não pôde ser executada por razões técnicas ou porque aquele não se revelou eficaz. Combinando essas técnicas, ocorreu controle da dor em $91,5 \%$ dos doentes; em $8,5 \%$ dos casos, a dor recorreu, embora com menor intensidade. Esses resultados são compatíveis com os de outros autores ${ }^{55,81,94}$. A neurectomia periférica é indicada para a confirmação diagnóstica, para o tratamento de doentes idosos debilitados ou com neuralgia bilateral, quando há anestesia de uma hemiface secundária à rizotomia contraleteral ${ }^{46}$. A neurólise da raiz trigeminal com glicerol aplicado no interior do gânglio de Gasser controla a dor em $88,9 \%$ a $98,7 \%$ dos casos e melhora em 1,3\%; a recorrência ocorre em $17,8 \%$ dos doentes durante os primeiros 2 a 48 meses, havendo necessidade de repetição do tratamento em $6,6 \%$. O resultado final é satisfatório em $86 \%$ das vezes. Sensação de dormência discreta associada à discreta hipoestesia ocorre em $60 \%$ dos doentes, lesões herpéticas em $50 \%$, analgesia em $84 \%$, disestesia em $18,6 \%$ e ceratite em $3,3 \%{ }^{36,100}$ dos doentes. A gangliólise com o glicerol é pouco empregada atualmente, pois a freqüência de recidivas é elevada e não se aplica a casos em que há lesão estrutural no gânglio de Gasser; parece ser útil principalmente no tratamento das algias da primeira divisão do nervo trigêmeo, em 
indivíduos que não apresentam condições clínicas para se submeterem a procedimentos operatórios de maior porte ${ }^{21,36}$. A rizotomia percutânea por radiofreqüência alivia a dor em $91 \%$ a $100 \%$ dos casos nas várias casuísticas $45,64,66,70,77,90,99,101,103,111,114$. Anormalidades da motricidade ocular extrínseca, disestesias, anestesia dolorosa, paralisia motora do nervo trigêmeo e ceratite neuroparalítica foram as complicações mais comuns. Ceratite ocorre em 1,4\% a $2,6 \%$ dos $\operatorname{casos}^{45,66,90,92}$, úlcera de córnea, em $1 \%$ a $2 \%{ }^{45,46,66,91,92}$, redução significativa da visão ou cegueira em $0,2 \%$ a $0,4 \%{ }^{90,102}$, parestesias em $8 \%$ a $10,9 \%{ }^{45,66,110,111,114}$, disestesias dolorosas em $0,5 \%$ a $5 \% 64,71,72,78,80,111,113$ e anestesia dolorosa em $0 \%$ a $5 \%{ }^{64,66,71,72,77,80,90,102,110,111,114,118}$. A recidiva da dor ocorre em $4 \%$ a $80 \%$ dos doentes ${ }^{45,57,64,65,66,71,77,90,97,102,110,111}$. O procedimento é seletivo e os resultados são consistentes e mantidos durante longo período de tempo, indicado também no tratamento da dor facial sintomática ${ }^{98,104,106,109}$. Shelden e cols. ${ }^{89}$ descreveram o tratamento da neuralgia do trigêmeo pela compressão do gânglio de Gasser, e Mullan e Lichtor ${ }^{67}$ descreveram a técnica de compressão percutânea do gânglio trigeminal. Os resultados imediatos foram excelentes em todos os doentes. Houve recorrência em 12\% dos casos, durante período que variou entre 6 e 54 meses. A compressão do gânglio trigeminal com balão é indicada em casos de neuralgia que acomete a primeira divisão do nervo e em doentes que não tenham condições de informar sobre a localização das parestesias induzidas pela estimulação induzida durante a rizotomia por radiofreqüência ${ }^{53}$. A radiocirurgia da raiz trigeminal é indicada quando não há condições clínicas ou anatômicas para a realização de procedimentos cirúrgicos percutâneos ou a céu aberto ${ }^{37}$. Os resultados são satisfatórios, mas o método é complexo e dispendioso. A nucleotratotomia trigeminal bulbar estereotáxica é utilizada no tratamento da dor craniofacial associada ao câncer ${ }^{18,19,43,106}$, neuralgia pósherpética ${ }^{85}$ e disestesias faciais que ocorrem após a lesão ou a rizotomia do nervo trigême ${ }^{44,85,86}$, da dor facial atípica e da neuralgia pós-herpética ${ }^{105}$. A descompressão neurovascular, descrita em 1959 por Gardner e Miklos ${ }^{33}$, e aperfeiçoada por Jannetta ${ }^{50}$, proporciona alívio da dor em $82,2 \%$ a $100 \%$ dos doentes à custa de mortalidade de até $4,3 \%$, paresia do VI nervo craniano, paralisia facial periférica ou ataxia cerebelar em outros $4,3 \%{ }^{26,68}$. É indicada em doentes jovens e em bom estado geral que optem pela preservação da sensibilidade facial.

Esses dados constituem elementos que enfatizam a importância dos procedimentos neurocirúrgicos funcionais como opção terapêutica importante no tratamento da neuralgia trigeminal, pois, além de aliviar a dor, apresentam morbidade reduzida ${ }^{14,15}$.

\section{Referências}

1. ALAJOUANINE T, THUREL R: La névralgie faciale. Importance du stimulus et de la zone d'exitation Déductions thérapeutiques et pathogénique. Nouv Presse Med 42:345-8, 1934.

2. ALVISI C, BORROMEI A: Varie modalità stiopatogenetiche della cosidetta nevralgia essenciale del nervo trigemio. Minerva Med 58:64-8, 1967.

3. AMELI NO: Avicenna and trigeminal neuralgia. J Neurol Sci 2:105-7, 1965.

4. APFELBAUM RI: A comparison of percutaneous radiofrequency neurolylis and microvascular decompression of the trigeminal nerve treatment of tic doloureux. Neurosurgery 1:16-21, 1977.

5. ASPESI N: Controle das trigeminalgias com O G 32883. Rev Bras Med 24:33-5, 1967.

6. BARRAQUER-BORDAS L: Sobre la herencia y etiopatogenia del tic doloroso trigeminal. Arq Neuropsiquiat (São Paulo) 7:241-63, 1949.

7. BEAVER DL, MOSES HL, GANOTE CE: Electron microscopy of the trigeminal ganglion. III. Trigeminal neuralgia. Arch Pathol 79:571-82, 1965.

8. BLOM S: Trigeminal neuralgia: its treatment with a new anticonvulsant drug (G-32883). Lancet 1:83940, 1962.

9. BLOM S: Tic doloureux treatd with a new anticonvulsant. Experiences with G-32883. Arch Neurol 9:285-90, 1963

10. BRAHAM J, SAIA A: Phenytoin in the treatment of trigeminal and other neuralgias. Lancet 2:892-3, 1960.

11. BURCHIEL KJ: Abnormal impulse geration in focally demyelinated trigeminal roots. J Neurosurg 53:67483, 1980.

12. BURKE WJG, GRANT JMF, SELBY G: The treatment of trigeminal neuralgia: a clinical trial of carbamazepina (“Tegretol"). Med J Aust 1:494-5, 1965.

13. BUSCAINO GA: Fisiologia della nevralgia essenziale del trigemino. Acta Neurol (Napoli) 35:137-44, 1980.

14. CORREAA CF, TEIXEIRA MJ: Balloon compression of the gasserian ganglion for the treatment of trigeminal neuralgia. Stereotact Funct Neurosurg 71:83-9, 1998.

15. CORREAA CF, TEIXEIRA MJ, OLIVEIRA JO JR, ROGANO LA: Microcompressão do gânglio de Gasser com balão Fogarty para o tratamento da neuralgia do trigêmeo. Arq Bras Neurocirurg 12:145-51, 1993.

16. COSTA AL: O G-32883 no tratamento sintomático da trigeminalgia. Arq Neuropsiquiat (São Paulo) 23:27982, 1965.

17. COURT JE, KASE CS: Treatment of tic doloureux with a new anticonvulsant (clonazepan). J Neurol Neurosurg Psychiatry 39:297-9, 1976.

18. CRUE BL, CARREGAL EJA, FELÖÖRY A: Percutaneous stereotacti radiofrequency trigeminal tractotomy with neurophysiological recordings. Confin Neurol 34:389, 1972.

19. CRUE BL, TOOD EM, CARREGAL EJA, KILHAM O: Percutaneous trigeminal tractotomy. Case report utilizing stereotactic radiofrequency lesion. Bull Los Angeles Neurol Soc 32:86-92, 1967.

20. CUSHING H: Major trigeminal neuralgics and their surgical treatment based on experiences with 332 gasserian operations; varieties of facial neuralgic. Am J Sc 160:157-64, 1920.

21. DADE LL, BENNERR MH: Percutaneous retrogasserian glycerol rhizotomy for tic doloureux: part I technic and results in 112 patients. Neurosurgery 14:424-30, 1984. 
22. DALLESSIO DJ: Trigeminal neuralgia. A pratical approach to treatment. Drugs 24:248-55, 1982.

23. DANDY WE: The treatment of trigeminal neuralgia by the cerebellar route. Ann Surg 96:787-95, 1932.

24. DANDY WE: Concerning cause of trigeminal neuralgia. Am J Surg 24:445-7, 1934

25. DECHAUME M: Temporomandibular joint pain (Syndrome of Costenhennebert-Dechaume). In Vinken PJ, Bruyn GW (ed): Handbook of Clinical Neurology. Amsterdam, North - Holland Publishing Company, 1968, Vol 5, pp 345-59.

26. DUPLEY J, CHIARELLI J, GRELLIER P, ROCHE JL, ROSSET MJ: Contribution à la chirurgie non mutilante de la névralgie-faciale essentielle. Neurochirurgie 20:593-8, 1974.

27. FORJAZ SV: Tratamento cirúrgico de trigeminalgia essencial. Arq Neuropsiquiat (São Paulo) 19:143-52, 1961.

28. FRAZIER CH: Bilateral trigeminal neuralgia. Ann Surg 100:770, 1934.

29. FRAZIER CH, RUSSEL EC: Neuralgia of the face. Arch Neurol Psychiatry (Chicago)11:557-63, 1924.

30. FROMM GH, CHATTA AS, TERRENCE CF, GLASS JD: Role of inhibitory mechanisms in trigeminal neuralgia. Neurology (NY) 31:683-7, 1981.

31. FROMM GH, TERRENCE CF, CHATTHA AF, GLASS JD: Baclofen in trigeminal neuralgia: its effect on the spinal trigeminal nucleus: a pilot study. Arch Neurol 37:768-71, 1980.

32. FURLOW LT: Bilateral trigeminal neuralgia. J Neurosurg 15:299-307, 1958.

33. GARDNER WJ, MIKLOS MV: Response of trigeminal neuralgia to "decompresion" of sensory root. Discussion of cause of trigeminal neuralgia. JAMA 170:1773-6, 1959.

34. GONZALEZ-REVILLA A: Tic doloureux and its relationship to tumors of the posterior fossa. Analysis of Twenty-four cases. J Neurosurg 4:233-9, 1947.

35. GRANT FC: Results of the operative treatment of mayor trigeminal neuralgia. Ann Surg 107:14-9, 1938.

36. HAKANSON S: Trigeminal neuralgia treated by injection of glycerol into the trigeminal cistern. Neurosurgery 9:638-46, 1981.

37. HANKANSON S, LEKSELL L: Stereotatic gamma radiation in trigeminal neuralgia. In 6th International congress of Neurological Surgery. Amsterdam, Excerpta Medica, 1977, pp 57.

38. HARRIS W: Bilateral tic. Its association with heredity and disseminated sclerosis. Ann Surg 103:161-72, 1936.

39. HARRIS W: An analysis of 1433 cases of parxysmal trigeminal neuralgia (trigeminal-tic) and the results of Gassrian alcohol injection. Brain 63:209-24, 1940.

40. HARRIS W: Rare forms of paroxysmal neuralgia and their relation to disseminated sclerosis. Brit Med J 2:1015-9, 1950

41. HÄRTEL F: Röentgenologische darstellung das foramen ovale des schädels und ihre bedertung für die behandlunmg der trigeminus neuralgie. Dtsch Med Wschr 61:1069-72, 1935.

42. HENDERSON WR: Trigeminal neuralgia: the pain and its treatment. $\mathrm{Br}$ Med 1:7-15, 1967.

43. HITCHCOCK E: Stereotactic trigeminal tractotomy. Ann Clin Res 2:131-5, 1970.

44. HITCHCOCK E: Stereotactic spinal surgery. In Carrea R, Levy D (ed): Neurological Surgery International Congress Series $n^{\circ} 433$. Amsterdam, Excepta Medica 1977, pp 271-80.
45. HITCHOCOCK E, TEIXEIRA MJ: A comparison of results from center-median and basal thalamotomies for pain. Surg Neurol 15:341-51, 1981.

46. HORRAX G: The role of minor procedures in the treatment of trigeminal neuralgia. Surg Clin North America 26:774-9, 1976

47. HORRAX G, POPPEN JL: Trigeminal neuralgia. Experiences with, and treatment employed in 468 patients during the past 10 years. Surg Gynec Obstet 61:394-402, 1935.

48. HOROWITHZ SH: Isolated facial numbers. Clinical significance and relation to trigeminal neuropathy. Ann Intern Med 80:49-53, 1974.

49. HUTCHINS LG, HARNSBERGER HR, HARDIN CW, DILLON WP, SMOKER WR, OSBORN AG: The radiologic assessment of trigeminal neuropathy. Am J Roentgenol 153:1275-82, 1989.

50. JANNETTA PJ: Arterial compression of the trigeminal nerve at the pons in patients with trigeminal neuralgia. J Neurosurg 26(Suppl):159-62, 1967.

51. JANNETTA PJ: Microsurgical approach to the trigeminal nerve for tic doloureux. Prog Neurol Surg 7: 180-200, 1976.

52. JANNETTA PJ: Treatment of trigeminal neuralgia by suboccipital and transtentorial cranial operations. Clin Neurosurg 24:538-49, 1977.

53. JEFFREY A, BROWN JA, MARK C, PREUL MC: Percutaneous trigeminal ganglion compression for trigeminal neuralgia. J Neurosurg 50:900-4, 1989.

54. KERR FW: Craniofacial neuralgias. In Bonica JJ, Liebeskind JC, Albe-Fessard DG (ed): Advances in Pain Research and Therapy. New York, Raven Press, 1979, pp 283-95.

55. KILLIAN JM, FROMM GH: Carbamazepine in the treatment of neuralgia. Use and size effects. Arch Neurol 19:129-36, 1968.

56. KING RB, MEAGHER JM: Studies of trigeminal nerve pontecials. J Neurosurg 12:393-402, 1955.

57. KIRSCHNER M: Zur Elektrochirurgie. Arch Klin Chir 167:761-8, 1931

58. KLINGHER M: Bévralgies vraies et frausses. Confin Neurol 25:330-2, 1965.

59. KUGELBERG E, LINDBLOM U: The mechanism of the pain in trigeminal neuralgia. J neurol Neurosurg Psychiatry 22:36-43, 1959.

60. LAZAR ML, KIRKPATRICK JB: Trigeminal neuralgia and multiple sclerosis: demonstration of the plaque in an operative case. Neurosurgery 5:711-17, 1979.

61. LEVINTHAL R, BENTSON JR: Detection of small trigeminal neurinomas. J Neurosurg 45:568-75, 1976.

62. LEWY FH, GRANT FC: Physiopathologic and pathonotomic aspects of major trigeminal neuralgia. Arch Neurol Psychiatry (Chicago) 40:1126-34, 1938.

63. MEIROWSKY AM, PIPITO FF: Surgical history of trigeminal neuralgia. Arch Neurol Psychiat 49:57480, 1943.

64. MENZEL J, PIOTROWISKI W, PENHOLZ H: Long term results of Gasserian Ganglion electrocoagulation. J Neurosurg 42:140-3, 1975.

65. MITCHELL AB, PARSON-SMITH BC: Trichloroethylene neuropathy. Brit Med J 1:422-43, 1909.

66. MOTTA P, DE SOUZA MTK, SENGUPTA RP: Radiofrequência - termocoagulação no tratamento da neuralgia do trigêmeo. Análise de 100 casos. Arq neuropsiquiatr (São Paulo) 38:33-44, 1980.

67. MULLAN S, LICHTOR T: Percutaneous microcompression of the trigeminal ganglion for trigeminal neuralgia. J Neurosurg 59:1007-12, 1983. 
68. NAESSEN PJ, WAELE LF: Microvascular decompression in the posterior fossa of the trigeminal nerve for tic doloureux and microvascular decompression of the facial nerve for hemifacial spasm. Acta Neurol Belg 80:95-8, 1980.

69. NAIDICH TP, LIN JP, LEEDS NE, KRICHEFF II, GEORGE AE, CLASE NE et al.: Computed tomography in the diagnosis of extra-axial posterior fossa masses. Radiology 120:333-9, 1976.

70. NUGENT GR, BERRY B: Trigeminal neuralgia treated by differential percutaneous radiofrequency coagulation of the Gasserian ganglion. J Neurosurg 40:517-23, 1974.

71. O'CONNEL JEA: Trigeminal false localizing signs and therir causation. Brain 101:119-42, 1978.

72. ONOFRIO BM: Radiofrequency percutaneous Gasserian ganglion lesions. Results in 140 patients with trieminal pain. J Neurosurg 42:132-39, 1975.

73. PATRICK H: The symptomatology of trifacial neuralgia. JAMA 62:1519-25, 1914.

74. PEET MM, SCHNEIDER RC: Trigeminal neuralgia. A review of six hundred and eighty nine cases with a follow-up study of sixty five percent of the group. J Neurosurg 9:356-77, 1952.

75. PENMAN J: The differential diagnosis and treatment of tic doloureux. Post Grad Med J 26:627-36, 1950.

76. PENMAN J: Trigeminal neuralgia. In Vinken PJ, Bruyn GW (ed): Handbook of Clinial Neurology. Amsterdam, North-Holland Publishing Company, 1968, Vol 5, pp 296-332.

77. PERTUISET B, PHILIPPON J, MACHANAKIAN A: Électrothermie rétro-gasseriene dans la névralgie faciale. Ann Med Int (Paris) 126:657-60, 1975.

78. RAND RW: Gardner neurovascular decompression of the trigeminal and facial nerves for tic doloureux and hemifacial spasm. Surg Neurol 16:329-32, 1981.

79. RASMUSSEN P, RÜSHED J: Facial pain treated with carbamazepin (Tegretol). Acta Neurol Scand 46:34080, 1970.

80. RISH BL: Cerebrovascular acident after percutaneous of thermocoagulation of the trigeminal ganglion. Case report. J Neurosurg 44:376-7, 1976.

81. ROCKLIFF BW, DAVIS EW: Controlled sequential trial of carbamezpine in the trigeminal neuralgia. Arch Neurol 15:129-36, 1966

82. RUGE D, BROCHNER R, DAVIS L: A study of the treatment of 637 patients with trigeminal neuralgia. J Neurosurg 15:528-36, 1958.

83. RUSHTON JG, McDONALD HNA: Trigeminal neuralgia: special considerations of nonsurgical treatment. JAMA 165:437-40, 1957.

84. RUSHTON JG, OLAFSON RA: Trigeminal neuralgia associated with multiple sclerosis: report of 35 cases. Arch Neurol 13:383-86, 1965

85. SCHVARCZ JR: Stereotactic trigeminal tractotomy. Confin Neurol 37:73-7, 1975.

86. SCHVARCZ JR: Spinal cord techniques: trigeminal nucleotomy and extralemniscal myelotomy. Appl Neurophysiol 41:99-112, 1978.

87. SEARLES RP, MLADINICH EK, MESSNER RP: Isolated trigeminal sensory neuropath: early manifestations of mixed connective tissue disease. Neurology (Minneap.) 28:1286-89, 1978.

88. SHEEFFER H, PELLAND M: Deux cas de névralgie trigéminale dans la syringobulbia. Le caractère de la douleur des les algies faciales d'origine centrale et leur traitment. Rev Neurol (Paris) 40:699-703, 1933.
89. SHELDEN CH, PUDENZ RH, FRESHWATER DB, CRUF $\mathrm{BL}$ : Compression rather than decompression for neuralgia. J Neurosurg 12:123-6, 1955.

90. SIEGFRIED J: 500 percutaneous thermocoagulation of the Gasserian ganglion for trigeminal pain. Surg Neurol 8:126-31, 1977.

91. SINDOU M: Thermocoagulation percutanée du trijumeau dans le traitment de la névralgie faciale essentialle. Résultats en function du siège de la thermolésion. Neurochirurgie 25:166-72, 1979.

92. SINDOU M, KERAVEL Y: La thermocoagulation percutanée du trijumeau. Noveau traitment neurochirurgical de la névralgie faciale essentialle. Nouv Press Med 5:1583-4, 1976.

93. SMIRNE S, SINATRA MG: II clonazepam nelle syndrome dolorose del distratto cefalico. Riv Neurol 49:140-50, 1979.

94. SPECULAND B, GASS AN, SPENCE ND, PILOWSKY I: Intractable facial pain and illness behavior. Pain 11:213-9, 1981

95. STÖHR M, PETRUCH F, SCHEGLMANN K: Somatosentory evoked potentials following trigeminal nerve stimulation in trigeminal neuralgia. Ann Neurol 9:636. 1981.

96. STOOKEY B, RANSOHOFF J: Trigeminal Neuralgia. Its History and Treatment. Springer, Charles C Thomas Publisher, 1959.

97. STOWSAND D, MARKAKIS E, LAUBNER P: Zur elektrokoagulation das Ganglion Gaserri bei der idiopathischen trigeminus neuralgie. Nervenartzt 44:44-7, 1973.

98. SWEET WH, WEPSIC JG: Controled thermocoagulation of trigeminal ganglion and rootlets for differential destruction of pain fibers. 1 Trigeminal neuralgia. J Neurosurg 40:143-56, 1974.

99. SWEET WH: Treatment of facial pain by percutaneous differential thermal trigeminal rhizotomy. Prog Neurol Surg 7:153-79, 1976.

100. SWEET WH, POLETTI CE, MACON JB: Treatment of trigeminal neuralgia and other facial pains by retrogasserian injection of glycerol. Neurosurgery 49:647-55, 1981

101. SWEET WH, WEPSIC JG: Relation of fiber size in trigeminal posterior root to conduction of impulses for pain and touch. Production of analgesia without anesthesia in the effective treatment of trigeminal neuralgia. Trans Am Neuro Assoc 95:134-9, 1970.

102. SWEET WH, WEPSIC JG: Controled thermocoagulation of trigeminal ganglion and rootlets for differential destruction of pain fibers. 1. Trigeminal neuralgia. J Neurosurg 40:143-56, 1974.

103. TAMBURUS P: Termocoagulação percutânea em neuralgia do trigêmeo. Arq Bras Neurocirurg 2:237 41, 1983.

104. TEIXEIRA MJ: A rizotomia percutânea por radiofreqüência e a descompressão vascular do nervo trigêmeo no tratamento das algias faciais. Dissertação de Mestrado, Faculdade de Medicina da Universidade de São Paulo. São Paulo, 1984, p. 425.

105. TEIXEIRA MJ: Facial pain - Various functional procedures for pain. In Gildenberg PL, Tasker RR (ed): Textbook of Stereotactic and Functional Neurosurgery. New York, McGraw-Hill Co, 1996, pp 1389-402.

106. TEIXEIRA MJ, AMARAL AC, LABRUNA R: Rizotomia percutânea por radiofreqüência no tratamento das algias otorrinofaringofaciais resultantes de tumores malignos. Arq Bras Neurocir 18:194-202, 1999. 
107. TEIXEIRA MJ, LIN TY, OLIVEIRA JR JO, VELASCO O, AGNER C, SHU EB et al.: Síndrome de Eagle. Arq Bras Neurocirurg 13:57-63, 1994.

108. TEIXEIRA MJ, OKADA M: Dores atípicas da face. In Siqueira JTT(ed): Dor Orofacial/ATM. Bases para o Diagnóstico Clínico. 2. Ed Curitiba, Editora Maio, 2001, pp 108-10.

109. TEW JM Jr, KELLER JT: The treatment of trigeminal neuralgia by percutaneous radiofrequency technique. Clin Neurosurg 12:557-78, 1976

110. TEW JM Jr, MAYFIELD FH: Trigeminal neuralgia. A new surgical approach (percutaneous electrocoagulation of the trigeminal nerve). Laryngoscope 83:1096-101, 1973.

111. THIRY S, ROTHERMANS JML: Traitment de la névralgie essentialle du trijumeau par stéreotaxie et electrocoagulation partielle sélective du ganglion de Gasser. Expérience portant sur 365 cas traités entre 1950 et 1970. Neurochirurgie 20:55-60, 1974.

112. THRUSH DC, SMALL M: How benign a symptom is facial numbness. Lancet 2:851-4, 1970.

113. TOOD EM, CRUE BL, CARREGAL EJA: Posterior percutaneous tractotomy and cordotomy. Confin Neurol 31:106-15, 1969.

114. TURNBULL IM: Percutaneous rhyzotomy for trigeminal neuralgia. Surg Neurol 2: 385-9, 1974.

115. VAN LOVEREN H, TEW JM, KELLER JT, MURRA MA: A 10 year experience in the treatment of trigeminal neuralgia. Comparison of percutaneous stereotactic rhizotomy and posterior fossa exploration. J Neurosurg 57:757-64, 1982

116. VER BRUGGHEN A: Paragasserian tumors. J Neurosurg 9:451-60, 1952.

117. WALKER E, MILES FC, SIMPSON RJ: Partial trigeminal rhizotomy using suboccipital approach. Experience in the treatment of trigeminal neuralgia. Arch Neurol Psychiatry 75:514-21, 1956.

118. WEPSIC JG: Complications of percutaneous surgery for pain. Clin Neurosurg 83:454-64, 1975.

119. WHITE JC, SWEET WH: Pain and the Neurosurgeon. Springfield, Charles C Thomas Publisher, 1969.

120. WILKINS H, SACHS E: Variations in skin anesthesia following subtotal ressection of posterior root, with a report of 20 cases illustrating a series of variations. Arch Neurol Psychiatry (Chicago) 29:19-49, 1933.

121. YOSHIMASU F, KURLAND LT, ELVELVACK LR: Tic doloureux in Rochester, Minnesota, 1945-1969. Neurology (Minneap.) 22:952-6, 1972.

Original recebido em setembro 2001

Aceito para publicação em outubro de 2001

\section{Endereço para correspondência:}

Clement Hamani

Rua Dr. Luiz Migliano, 761 - ap. $142 c$

CEP 05711-001 - São Paulo, SP

E-mail:chamani@uol.com.br 